\title{
Acute Urinary Retention: A Rare Presentation in Acute Myeloid Leukemia
}

\author{
Barik $S^{1}$, Sinha $S^{2}$ \\ ${ }^{1}$ Dr Sumit Barik, Specialist and designated Associate Professor, Department of Pathology and Hematology, ${ }^{2}$ Dr Sweta \\ Sinha, Senior Resident, Department of Transfusion Medicine. Both are affiliated with ESI Post Graduate Institute of \\ Medical Science and Research, New Delhi. 110015, India
}

Address for correspondence: Dr. Sweta Sinha, Email: sweta20eleven@gmail.com

\begin{abstract}
Hyperleukocytosis is defined when the total leukocyte count is more than $100000 / \mathrm{cmm}$. Leukostasis occurs in hyperleukocytic acute myeloid leukemia when the numbers of blasts are very high. Leukostasis causes impaired normal functioning of vascular systems because of crowding of blasts in its microvasculature or in its vessels supplying the nerves. Leukostasis causes early deaths in acute myeloid leukemia (AML) due to involvement of central nervous system and lungs. Acute retention of urine because of haematological causes is rarely seen. Dysfunction of bladder because of leukostasis in hyperleukocytic AML is warning. Cytoreduction therapy in hyperleukocytic AML prevents early deaths.
\end{abstract}

Key words: Acute myeloid leukemia, hyperleukocytosis, leukostasis, Urinary retention.

\section{Introduction}

Hematological malignancies like acute leukemias and chronic leukemias can produce organ or system disorder because of leukostasis. $5-30 \%$ cases of acute leukemia present because of hyperleukocytosis with a total leukocyte count of more than $100000 / \mathrm{cmm}$ [1]. The microcirculation of the nervous system, lungs, eye and penis is most sensitive to the effects of leukostasis. Dizziness, stupor, dyspnea, and priapism may occur [2]. Cerebral haemorrhages and respiratory failure are grievous complications of hyperleukocytic AML.

The mortality in hyperleukocytic AML is very high and management of hyperleukocytosis is of utmost importance in saving the patient. The leukapharesis and chemotherapy is targeted towards faster reduction in counts and correcting abnormality produced by leukostasis [3].

Urinary retention because of leukostasis as presentation in acute myeloid leukemia is rare. Prompt intervention with aim of leukoreduction for relieving urinary retention prevented other possible complications of leukostasis in this patient.

\section{Case Report}

A girl of 10 years age was brought by parents in emergency department with complaints of pain in abdomen and inability to pass urine since last two days.
She also complained of weakness and bodyache. There was no past history of injury, fever, seizures or unconsciousness. On examination, bladder was palpable till hypogastrium. Gynaecological examination ruled out any abnormal finding pertaining to female genital system.

Pallor was evident but no icterus and cyanosis was found. Liver was not palpable though spleen was palpable just below costal margins. Any other significant finding could not be elicited in systemic examination. Ultrasound examination confirmed mild increase in spleen but any other abnormality was not detected. Urine examination was sent after evacuating the bladder with catheter.

Albumin and sugar were absent in urine and microscopic examination showed 1 to 2 pus cell per high power field.

Any other abnormality was not found in urine examination. Hemogram was sent to the department of hematology for cell counts and type of anemia. Hemoglobin was found to be $5.9 \mathrm{gm} \mathrm{\%}$, total leukocyte count was 1,50, $000 / \mathrm{cmm}$ and platelets were 1,60, 000 /cmm. Peripheral smear examination showed blasts making 95\%, rest were lymphocytes and neutrophils. Platelets were adequate and no parasite was seen. A diagnosis of acute myeloid leukemia with hyperleukocytosis was made.

Available online at: www.ijmrr.in 267 | P a g e 


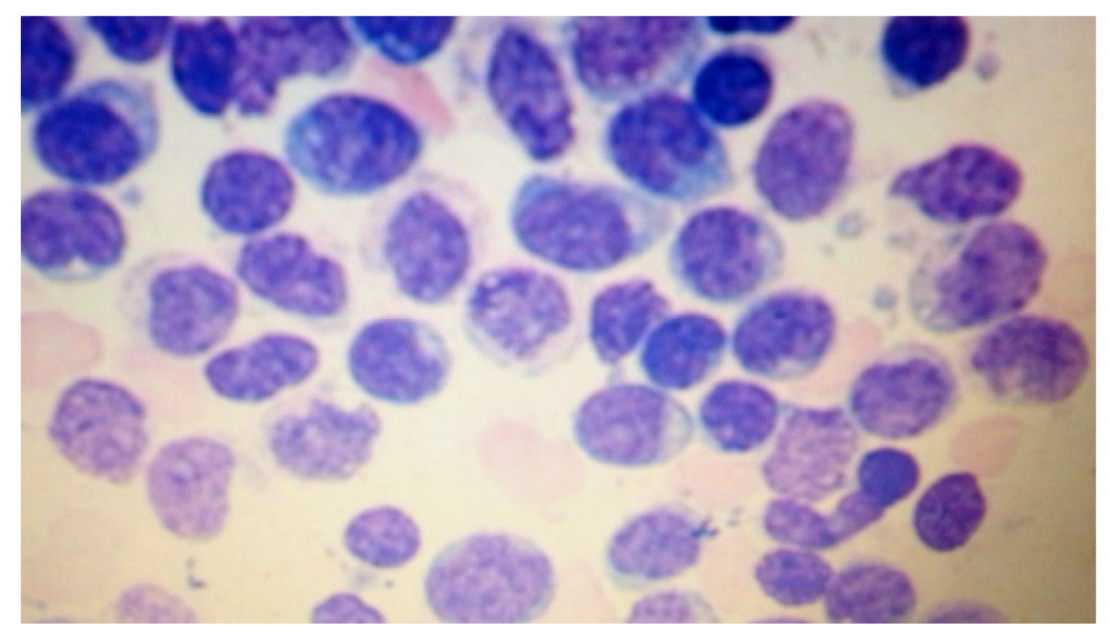

Case Report

Figure 1: Hyperleukocytic Acute myeloid leukemia (M4). (Magnification 100x10x). Myeloblasts and monoblasts are distinctly seen.

Cytochemistry confirmed acute myelomonocytic leukemia (M4 variety according to FAB classification). Imaging studies did not show any compressing lesion or a tumour aggregate (myeloid sarcoma or chloroma) which could explain bladder dysfunction. A presumptive diagnosis of Hyperleukocytic AML with Leukostasis was made and patient was sent for leukoreduction. Leukapharesis was done and the leukocyte counts were brought down to $80000 / \mathrm{cmm}$. The function of urinary bladder corrected and patient was able to pass urine. Definitive induction therapy was started and other measures like hydration and alkalinisation were done as a regime for leukoreduction. Urinary retention never occurred again till her stay in hospital and she was discharged with follow up instructions.

\section{Discussion}

Our patient was diagnosed with hyperleukocytic AML causing bladder retention. Her leukocyte counts were $1,50000 / \mathrm{cmm}$ and blasts were $95 \%$. The blasts were myelomonocytic. Hyperleukocytosis is commonly seen in myelomonocytic AML. The symptoms of hyperleukocytosis are because of leukostasis in the blood vessels supplying the organ or system. A very high percentage of circulating blasts with a very high total count has been associated with leukostasis. Overcrowding of leukemic blasts in the capillaries of the microcirculation and effects of soluble cytokines are reason for the development of leukostasis. Leukemic cell's ability to respond to chemo tactic cytokines and their expression of specific adhesion molecules are probably more important in determining whether leukostasis will develop than the number of circulating blasts [4]. Our patient came with acute retention of urine.
She had hyperleukocytic AML of M4 variety. Leukostasis is more commonly associated with myeloid than lymphoid leukemias because of the increased size and rigidity of myeloblast. The arbitrary threshold of leukocyte counts is $1,00,000 / \mathrm{cmm}$ in acute leukemias, to produce effects of leukostasis. However chronic leukemias usually require the counts more than 3 , $00,000 / \mathrm{cmm}$ to produce effects of leukostasis [2].

Our patient developed dysfunction of urinary bladder because of leukostasis. Acute limb ischemia, and renal vein thrombosis and aortic occlusion have all been described with leukostasis and acute hyperleukocytosis [5]. Uncommonly leukostasis can occur in bladder neck, testes, penis rarely vulva [2]. Our patient had not shown any signs of bleeding and her platelet counts were adequate. The imaging studies ruled out myeloid sarcoma or any other lesion which could explain bladder dysfunction. Hyperleukocytic AML, absence of bleeding and no evidence of myeloid sarcoma in imaging studies have led us to diagnose leukostasis. Leukostatic effect on vascular or nerve supply of bladder caused dysfunction of bladder. Marbello et al found very high mortality in hyperleukocytic AML compared to those who had counts less than $1,00,000 / \mathrm{cmm}$. The major cause had been intracranial haemorrhage and pulmonary failure [6]. Background knowledge of such possibility and availability of leukoreduction therapy in the form of leukapheresis and chemotherapy can prevent early death in AML [7]. Leukapharesis was advised with an aim of correcting bladder dysfunction and prevention of intracranial and pulmonary effects of leukostasis. Induction chemotherapy was started and the patient was discharged after substantial improvement. Cytoreduction by leukaphersesis and chemotherapy could correct the 
bladder dysfunction produced by leukostasis of hyperleukocytic AML.

\section{Conclusion}

Acute retention of urine can be a presenting feature in hyperleukocytic AML because of leukostasis. This condition should be thought when features like anemia and bone pain is associated with retention of urine. Cytoreduction therapy should be considered whenever leukocyte count in AML is more than 1,00,000/cmm in association with bladder dysfunction or any other organ dysfunction. This reduces mortality in AML.

\section{References}

1. Porcu P, Farag S, Marcucci G, Cataland SR, Kennedy MS, Bissell M. Leukocytoreduction for acute leukemia. Ther Apher 2002; 6:15-23.

2. Lichtman MA. Classification and clinical manifestations of clonal myeloid disorders .In: Kaushansky K, Lichtman MA, Beutler E, Kipps TJ, Seligsohn U, Prchal JT, editors. Williams Hematology $.8^{\text {th }}$ ed, US: Mc Graw -Hill and company; 2010: p. 121123.
3. Ganzel C, Becker J, Mintz PD, Lazarus HM, Rowe JM. Hyperleukocytosis, leukostasis and leukapheresis: practice management.Blood Rev.2012May;26(3):117-22

4. Porcu P, Cripe LD, Ng EW, Bhatia S, Danielson CM, Orazi A, Mc Carthy LJ. Hyperleukocytic leukemias and leukostasis: a review of pathophysiology, clinical presentation and management. Leuk Lymphoma. 2000; 39:1-18.

5. Majhail NS, Lichtin A. Acute leukemia with a very high leukocyte count: Confronting a medical emergency. Cleveland Clinic Journal of Medicine, 2004; 71(8):633-7

6. Marbello L, Ricci F, Nosari AM, Turrini M ,Nador G , Nichelatti M ,Tedeschi A, Vismara E ,Morra E. Outcome of hyperleukocytic adult acute myeloid leukaemia: a single-center retrospective study and review of literature .Leuk Res. 2008 Aug;32(8):1221-7.

7. Bug G, Anargyrou K, Tonn T, Bialleck H, Seifried E, Hoelzer D, Ottmann OG. Impact of leukapheresis on early death rate in adult acute myeloid leukemia presenting with hyperleukocytosis. Transfusion 2007 Oct;47(10):1843-50.

How to cite this article?

Barik S, Sinha S. Acute Urinary Retention: A Rare Presentation in Acute Myeloid Leukemia. Int J Med Res Rev 2014;2(3):267-269. doi:10.17511/ijmrr.2014.i03.18 